\title{
Identification of new biomarker candidates for glucocorticoid induced insulin resistance using literature mining
}

Wilco WM Fleuren ${ }^{1,2}$, Erik JM Toonen ${ }^{3}$, Stefan Verhoeven ${ }^{4}$, Raoul Frijters ${ }^{1,6}$, Tim Hulsen ${ }^{1,7}$, Ton Rullmann ${ }^{5}$, René van Schaik ${ }^{4}$ Jacob de Vlieg ${ }^{1,4}$ and Wynand Alkema ${ }^{1,8^{*}}$

\author{
* Correspondence: \\ w.alkema@cmbi.ru.nl \\ ${ }^{1}$ Computational Drug Discovery \\ (CDD), CMBI, NCMLS, Radboud \\ University Nijmegen Medical Centre, \\ P.O. Box $91016500 \mathrm{HB}$, Nijmegen, \\ The Netherlands \\ ${ }^{8}$ Present address: NIZO Food \\ Research BV, Ede, The Netherlands \\ Full list of author information is \\ available at the end of the article
}

\begin{abstract}
Background: Glucocorticoids are potent anti-inflammatory agents used for the treatment of diseases such as rheumatoid arthritis, asthma, inflammatory bowel disease and psoriasis. Unfortunately, usage is limited because of metabolic sideeffects, e.g. insulin resistance, glucose intolerance and diabetes. To gain more insight into the mechanisms behind glucocorticoid induced insulin resistance, it is important to understand which genes play a role in the development of insulin resistance and which genes are affected by glucocorticoids.

Medline abstracts contain many studies about insulin resistance and the molecular effects of glucocorticoids and thus are a good resource to study these effects.

Results: We developed CoPubGene a method to automatically identify gene-disease associations in Medline abstracts. We used this method to create a literature network of genes related to insulin resistance and to evaluate the importance of the genes in this network for glucocorticoid induced metabolic side effects and anti-inflammatory processes.

With this approach we found several genes that already are considered markers of GC induced IR, such as phosphoenolpyruvate carboxykinase (PCK) and glucose-6phosphatase, catalytic subunit (G6PC). In addition, we found genes involved in steroid synthesis that have not yet been recognized as mediators of GC induced IR.
\end{abstract}

Conclusions: With this approach we are able to construct a robust informative literature network of insulin resistance related genes that gave new insights to better understand the mechanisms behind GC induced IR. The method has been set up in a generic way so it can be applied to a wide variety of disease networks.

Keywords: Literature mining, Insulin resistance, Glucocorticoids, Gene networks

\section{Background}

Glucocorticoids (GCs) are often prescribed for the treatment of inflammatory diseases such as rheumatoid arthritis, asthma, inflammatory bowel disease and psoriasis [1-3]. Despite their excellent efficacy, usage is limited because of side-effects such as insulin resistance, glucose intolerance, diabetes, central adiposity, dyslipidemia, skeletal muscle wasting and osteoporosis [4-8].

GCs bind to the glucocorticoid receptor (GR), which then dimerizes and translocates to the nucleus where it influences gene transcription. Positive regulation of genes

(c) 2013 Fleuren et al.; licensee BioMed Central Ltd. This is an Open Access article distributed under the terms of the Creative Commons Attribution License (http://creativecommons.org/licenses/by/2.0), which permits unrestricted use, distribution, and reproduction in any medium, provided the original work is properly cited. 
(transactivation) is mainly mediated by direct binding of the GR-GC complex to glucocorticoid response elements located in the regulatory region of a target gene. The GR-GC complex may also bind to negative glucocorticoid response elements, which leads to a negative regulation of genes (transrepression). It is believed that transrepression, in which proinflammatory genes are downregulated, is mainly responsible for the efficacy of GCs as anti-inflammatory drugs [5,7], while transactivation might be responsible for the GC-induced adverse effects [9].

An important side effect is the development of insulin resistance (IR), because it is the onset of many metabolic diseases and conditions such as obesity, diabetes mellitus and hypertension. IR is a physiological condition in which a given concentration of insulin produces a less-than-expected biological effect. These biological effects are different depending on the tissue in which they occur. For instance, under IR conditions, fat and muscle cells fail to adequately respond to circulating insulin, which results in reduced glucose uptake, and subsequently higher glucose levels in blood [10,11]. In liver cells the IR- effects can be seen in reduced glycogen synthesis and storage, and a failure to suppress glucose production and release into the blood.

One way by which GCs induce IR is by inhibition of the recruitment of GLUT4 glucose transporter, which results in reduced insulin-stimulated glucose transport in skeletal muscle [12]. However, not all mechanisms involved in GC-induced side effects are not completely understood. To gain more insight into mechanisms behind GC induced IR, it is important to understand which genes play a role in the development of insulin resistance and which genes are affected by GCs.

It has been widely recognized that a system approach in which networks of genes in their functional context are studied, contributes to a better understanding of the mechanisms and pathways related to the disease and the drug effects [13-17]. To study a gene network related to a disease such as IR, a list of disease related genes as well as a notion of the interactions between these genes is needed.

Literature databases such as Medline contain many studies about IR and the molecular effects of synthetic glucocorticoids and thus are a good resource that can be used to create and study disease related gene networks.

The retrieval of relevant gene-disease associations out of the millions of abstracts in Medline is very labor intensive and thus a text mining system is needed to this in an automated fashion.

In previous work we reported about CoPub [18-20], a publicly available text mining system, which has successfully been used for the analysis of microarray data and in toxicogenomics studies [21-26]. CoPub calculates keyword co-occurrences in titles and abstracts from the entire Medline database, using thesauri for genes, diseases, drugs and pathways. We used this technology to develop CoPubGene, a rapid gene - disease network building tool. To evaluate the importance of genes in these networks we implemented a method to score the importance of genes in biological processes of interest by incorporating their functional neighborhood.

We used CoPubGene to create a network of genes related to insulin resistance and to evaluate the importance of the genes in this network for glucocorticoid induced metabolic side effects and anti-inflammatory processes.

By using this method, we identified several genes that already are considered markers of GC induced IR, such as phosphoenolpyruvate carboxykinase (PCK) and glucose-6- 
phosphatase, catalytic subunit (G6PC) $[27,28]$. Even more importantly, we were able to identify genes involved in steroid synthesis that have not yet been recognized as mediators of GC induced IR.

\section{Methods}

\section{CoPubGene}

We constructed CoPubGene as a SOAP based web service (Table 1). This CoPub Web Service WSDL is created in Eclipse using the so-called Document Literal Wrapped style. The web service provider code is written in Perl using the SOAP::WSDL module and is available via the CoPub portal http://www.copub.org.

\section{Retrieval of Gene-Disease associations}

To create disease related gene networks, we used CoPubGene to retrieve gene-disease and gene-gene associations from Medline abstracts. Disease terms which had significant gene associations based on the R-scaled score ( $\mathrm{rs}>35$ ) and literature count (lc $>5$ ) in Medline abstracts, were extracted from the CoPub thesaurus.

Table 1 List of available operations of the CoPub Web Service

\begin{tabular}{|c|c|c|c|c|}
\hline Name & Operation name & Input & Output & Description \\
\hline Get genes & Get_genes & $\begin{array}{l}\text { Gene } \\
\text { name, } \\
\text { gene } \\
\text { identifier }\end{array}$ & $\begin{array}{l}\text { Biological identifier } \\
\text { (s), with gene } \\
\text { specific } \\
\text { information }\end{array}$ & $\begin{array}{l}\text { Each gene in CoPub belongs to an } \\
\text { internal identifier (biological identifier). } \\
\text { Get_genes converts the input gene to } \\
\text { such a Biological identifier. This } \\
\text { biological identifier serves as an input } \\
\text { for subsequent operations. }\end{array}$ \\
\hline $\begin{array}{l}\text { Get } \\
\text { Keywords }\end{array}$ & Get_keywords & Keyword & $\begin{array}{l}\text { Biological identifier } \\
\text { (s), with keyword } \\
\text { specific } \\
\text { information }\end{array}$ & $\begin{array}{l}\text { Retrieves for a set of keywords, the } \\
\text { Biological identifiers to which these } \\
\text { keywords belong in CoPub. These } \\
\text { biological identifiers serve as an input } \\
\text { for subsequent operations. }\end{array}$ \\
\hline $\begin{array}{l}\text { Get } \\
\text { references }\end{array}$ & Get_references & $\begin{array}{l}\text { Biological } \\
\text { identifier } \\
\text { (s) }\end{array}$ & $\begin{array}{l}\text { Literature } \\
\text { references }\end{array}$ & $\begin{array}{l}\text { Given a Biological identifier, retrieves } \\
\text { all abstracts in which the term occurs. }\end{array}$ \\
\hline $\begin{array}{l}\text { Get } \\
\text { literature } \\
\text { neighbours }\end{array}$ & Get_literature_neighbours & $\begin{array}{l}\text { Biological } \\
\text { identifier } \\
\text { (s) }\end{array}$ & $\begin{array}{l}\text { Literature } \\
\text { neighbors }\end{array}$ & $\begin{array}{l}\text { Given a Biological identifier, retrieves a } \\
\text { list of keywords which are mentioned } \\
\text { in the literature together with the } \\
\text { input term. }\end{array}$ \\
\hline $\begin{array}{l}\text { Get } \\
\text { enriched } \\
\text { keywords }\end{array}$ & Get_enriched_keywords & $\begin{array}{l}\text { List of } \\
\text { gene } \\
\text { identifiers }\end{array}$ & $\begin{array}{l}\text { List of enriched } \\
\text { keywords }\end{array}$ & $\begin{array}{l}\text { For a list of genes, this operation } \\
\text { calculates a keyword } \\
\text { overrepresentation. }\end{array}$ \\
\hline $\begin{array}{l}\text { Get } \\
\text { literature } \\
\text { network }\end{array}$ & Get_literature_network & $\begin{array}{l}\text { Biological } \\
\text { identifier } \\
\text { (s) }\end{array}$ & $\begin{array}{l}\text { SVG / Cytoscape } \\
\text { network }\end{array}$ & $\begin{array}{l}\text { For a set of genes, the operation } \\
\text { creates a network of genes. }\end{array}$ \\
\hline $\begin{array}{l}\text { Get } \\
\text { categories }\end{array}$ & Get_Categories & - & List of categories & $\begin{array}{l}\text { Returns a list of categories of terms in } \\
\text { CoPub }\end{array}$ \\
\hline Get chips & Get_chips & - & List of microarrays & $\begin{array}{l}\text { Returns a list of available Affymetrix } \\
\text { chip names in CoPub. }\end{array}$ \\
\hline Version & Version & - & $\begin{array}{l}\text { Version of code } \\
\text { and literature }\end{array}$ & $\begin{array}{l}\text { Returns the version of the code and } \\
\text { literature. }\end{array}$ \\
\hline Selftest & Selftest & - & $\begin{array}{l}\text { Diagnostic } \\
\text { information }\end{array}$ & - \\
\hline
\end{tabular}

Biological identifiers are used by CoPub to identify biological concepts in the system. Each biological concept has a unique identifier. 


\section{Disease clustering}

Disease clustering was done in $\mathrm{R}$ (http://www.r-project.org) using the pvclust $\mathrm{R}$ package with "complete" setting for hierarchical clustering, based on correlation distance of R-scaled scores between genes and diseases, with 100 bootstrap replications. The hierarchical cluster was visualized using Denroscope [29]. Additional gene set enrichment analysis against the GENETIC_ASSOCIATION_DB_DISEASE was done with the annotation server DAVID [30,31].

\section{Creation of IR gene network}

CoPubGene was used to create a set of genes related to IR, by searching for associations between genes and IR in Medline abstracts using default values ( $\mathrm{rs}>30$ and $\mathrm{lc}>$ 5). Subsequently the IR-gene network was created by connecting genes that had significant co-occurrences with each other.

\section{Keyword enrichment analysis of IR related genes}

Keyword enrichment analysis on the list of IR related genes was done against disease and drug terms from the CoPub database. Threshold values were chosen using default values.

\section{Analysis of the IR gene network and calculation of neighbor score for genes}

The IR gene network was analyzed by mapping specific occurrences of the IR related genes with 'inflammation' and 'dexamethasone' in Medline abstracts onto the network. For the evaluation of the involvement of a gene, calculation of the literature score for a given gene and a given disease term, also the effects of dexamethasone and inflammation on the connecting genes are included. The literature score for gene $g$ with term $d$ is calculated in the following way:

$$
\text { Literature }_{\text {score }} g=\frac{g 1+N s}{2}, N s=\frac{(r g 2 * g 2)+(r g 3 * g 3)+. .(r g n * g n)}{r g 2+r g 3+. . r g n}
$$

In which $g 1$ is the R-scaled score of gene $g$ with term $d$, and Ns is the literature score of its neighboring genes with term $d$. This latter score $N s$ is calculated using the Rscaled score of each neighboring gene of gene $g$ with term $d$ (g2, g3,..,gn) relative to its relation (R-scaled score) with gene $g$ (rg2, rg3,..,rgn).

\section{Results}

We developed CoPubGene by creating a number of web service operations that can be used to construct networks of genes based on their co-occurrences in Medline abstracts. These web service operations can be combined to answer a variety of biological questions (Table 1). For example, the question "to what biological processes is this gene related?" can be answered by running the "get genes" and "get literature neighbours" functions. Using subsequently the "get references" function will return all the relevant pubmed entries in which the gene and keywords co-occur. By applying the "get keywords" and "get literature neighbours" functions one can retrieve all disease terms that are linked to a given drug term in the Medline abstract, or vice versa, retrieve all drug terms that are linked to a given disease term in abstracts. The networks that are created can be written to Cytoscape for downstream applications and visualizations. Also more advanced questions such as the 
construction of disease related gene networks, and subsequent calculation of keyword enrichment in this network can be addressed in an automatic way. In Table 1 the available web service operations are shown.

\section{Retrieval of gene-disease associations}

Our aim was to get insight into the pathways and genes that are involved in insulin resistance, and the effect of glucocorticoids on this network. As a first step we created a list of genes associated with insulin resistance using CoPubGene. This yielded a list of 384 genes each of them connected to IR with an $\mathrm{R}$ scaled score (in Additional file 1: Table S2A the top scoring genes with IR are shown, the full list is available in Additional file 2: Table S2). To evaluate the quality of this list and to investigate whether this gene list is unique for IR or whether this list contains a large number of genes that are associated with multiple diseases we constructed a gene association list for all diseases in the disease thesaurus of CoPub, using similar parameter settings as used for construction of the IR gene list. This yielded a list of disease profiles with for each disease, a number of genes connected to that disease with an $\mathrm{R}$ scaled score. (Additional file 1: Table S2 shows the results for a few selected diseases, the full table is available in Additional file 2: Table S3).

These disease profiles were clustered using hierarchical clustering with multiscale bootstrap resampling, grouping together disease terms which have a similar profile, i.e. co-occur with the same genes (Figure 1; See Additional file 3: Figure S2 for the cluster with all bootstrap values). It appeared that a number of clusters of similar disease terms i.e. disease terms for which it is known that they have similar symptoms or have a similar mode of action, could be identified. For instance cancer related terms, such as 'cancer of breast', 'cancer of prostate' and 'colon cancer' are clustered together and inflammatory related disease terms such as 'psoriasis', 'inflammatory bowel disease' and 'asthma' are clustered together. These clusters also have high unbiased (AU) bootstrap values, indicating strong evidence for these clusters. To further confirm that the found gene-disease associations by CoPub are indeed biologically relevant, for each subcluster in Figure 1, we collected the union of all genes for that sub-cluster, and used these genes to perform a functional annotation analysis against the genetic association disease database using DAVID. The results of this analysis indicated that indeed similar disease terms to CoPub were found by DAVID (for the results of this analysis see Additional file 4). These analyses showed that with CoPubGene we are able to construct a relevant list of specific IR related genes that can be used for further analysis and that CoPubGene can be used to create a variety of disease related genes lists.

\section{Network of insulin resistance related genes}

To create the IR gene network, we used the 384 genes from the IR gene list and connected the genes based on their co-occurrences with each other in Medline abstracts. The resulting network is shown in Figure 2A. We found that 381 genes of the IR gene list were connected to at least one other gene. We identified a number of hubs such as peroxisome proliferator-activated receptor gamma (PPARG), insulin receptor substrate 1 (IRS1), v-akt murine thymoma viral oncogene homolog 1 (AKT1), insulin receptor (INSR), solute carrier family 2 (facilitated glucose transporter), member 4 (SLC2A4) and 


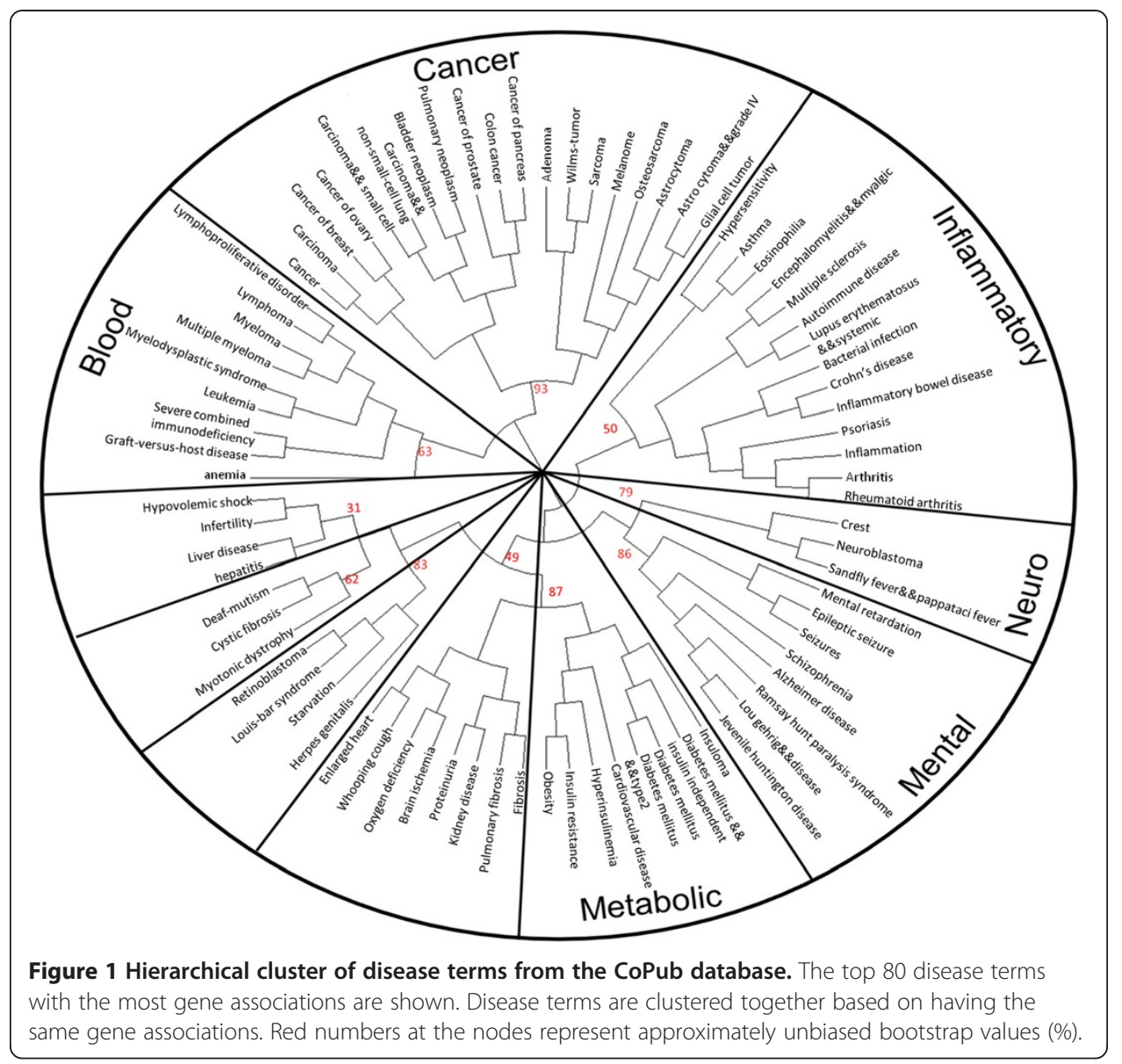

insulin (INS) which were connected to more than 100 other genes. The resulting network is a scale free network, as indicated by the distribution of connectivity that follows a power law distribution which is indicative for a scale free network (Additional file 5: Figure S1) [32]. Although the above network has the characteristics of a biological network, and contains the expected genes as central hubs, without additional annotations this network representation is still largely uninformative and contains too little substructure to draw biological conclusions.

\section{Annotation of the network with drugs and diseases terms}

As a first step towards annotating the network and identification of sub networks with a shared biological function, we investigated which drugs and diseases in the literature are specifically linked to this network using a keyword enrichment analysis on the list of IR related genes (For details about the enrichment method see Table 1). This enrichment yielded a number of drugs that are known drugs for the treatment of diabetes such as 'rosiglitazone,' 'metformin', 'pioglitazone', and also 'glucagon' and 'insulin' which are frequently used for the treatment of hypoglycemia and hypoinsulinemia (Table 2A. For the full list see Additional file 2: Table S4A). Notably, among these top scoring drugs we found dexamethasone, a well known synthetic glucocorticoid. High scoring genes with dexamethasone are for instance CEBPA, SERPINA6, PCK2 and GPD1 (for a 
full list of genes per enriched drug term, see Additional file 2: Table S4A.2), which also have been mentioned in the development of several metabolic diseases [33-37].

There are several top scoring over-represented terms that are related to metabolic diseases, e.g. 'diabetes mellitus', 'obesity', 'diabetes mellitus, type 2' and 'hyperinsulinemia' (Table $2 \mathrm{~B}$ ). The fact that these terms are high scoring is expected since we constructed the gene network based on the keyword insulin resistance. However we also found diseases that share a common origin with insulin resistance such as cardiovascular disease (Table 2B). The most interesting high scoring term for our particular research question was the nonmetabolic term 'inflammation', which was represented in the network by genes such as IL6, IL18, IL1RA, SOCS1, SOCS3, CCL2 and CCR2. Several of these genes have been mentioned in studies to be involved in the development of metabolic diseases. For instance, elevated levels of IL6 in subjects with obesity and diabetes showed an association between insulin resistance and IL6 [38]. Studies in mice showed that CCR2 deficiency or antagonism of this receptor resulted in attenuation of systemic insulin resistance and development of obesity, hence suggesting a modulating role of CCR2 in this [39,40].

These results show that even with an unbiased data driven construction of a gene network, the relation between IR, dexamethasone and inflammation is discovered based on the genes that play a role in these effects. We subsequently highlighted the genes in the IR network that are related to inflammation and dexamethasone (Figure 2).

\section{Genes linked to inflammation and glucocorticoids in the context of insulin resistance}

From a drug development perspective it is interesting to separate the desired effect of GCs on inflammatory processes from the undesired effect on metabolic processes. To
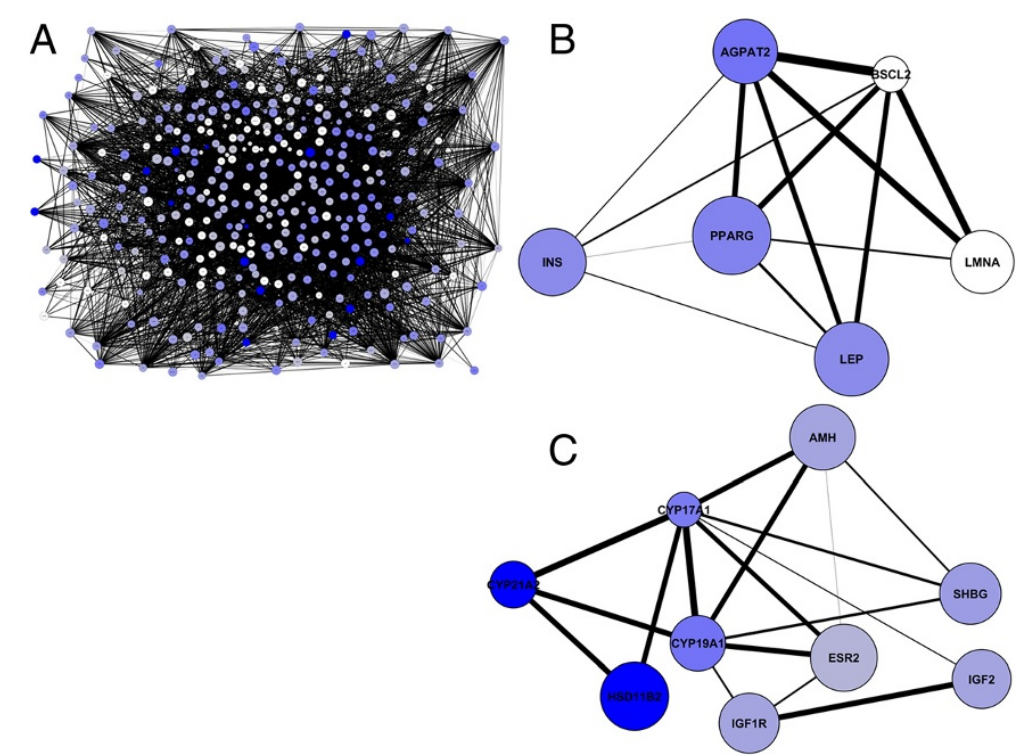

Figure $\mathbf{2}$ Literature network of insulin resistance related genes (A). Genes, represented by nodes are linked, based on co-occurrences in Medline abstracts. The thickness of the edge indicates the strength of the link between two genes (R-scaled score). Genes in blue have a co-occurrence with dexamethasone in Medline abstracts (R-scaled score). The strength of the link with dexamethasone is given by the color shading, ranging from no link (white) to a strong link (dark blue). The strength of the link with inflammation (R-scaled score) is given by the size of the node of the gene, ranging from no link (normal size of the node) to a strong link with inflammation (large size of the node). Sub-network for gene PPARG (B). Sub-network of Cytochrome P450s (C). 
Table 2 Over-represented drug and disease terms ( $P$-value $<0.05)$

\begin{tabular}{|c|c|}
\hline$A$ & \\
\hline Term & Number of genes \\
\hline insulin & 358 \\
\hline dexamethasone & 195 \\
\hline nitric oxide & 193 \\
\hline estrogen & 169 \\
\hline adenosine & 151 \\
\hline estradiol & 145 \\
\hline rosiglitazone & 125 \\
\hline actinomycin & 124 \\
\hline actinomycin d & 121 \\
\hline glucagon & 120 \\
\hline thrombin & 108 \\
\hline progesterone & 97 \\
\hline trypsin & 86 \\
\hline nicotinamide & 85 \\
\hline metformin & 84 \\
\hline pioglitazone & 82 \\
\hline B & \\
\hline Term & Number of genes \\
\hline insulin resistance & 381 \\
\hline obesity & 263 \\
\hline inflammation & 219 \\
\hline diabetes mellitus & 190 \\
\hline cardiovascular disease & 181 \\
\hline Diabetes mellitus,type 2 & 173 \\
\hline Oxygen deficiency & 164 \\
\hline fibrosis & 138 \\
\hline hyperinsulinemia & 137 \\
\hline Cancer of breast & 131 \\
\hline Adiposity & 130 \\
\hline cancer & 128 \\
\hline starvation & 120 \\
\hline
\end{tabular}

The top scoring drug terms in the IR network from the CoPub database (A). Top scoring disease terms from the CoPub database in the IR network (B).

rank each gene with respect to the relation with GC and inflammation, we calculated for each gene a literature score with dexamethasone and inflammation. Subsequently we focused on genes that score low on inflammation and high on dexamethasone (Figure 3). These genes are thought to be more exclusively related to GC induced IR. For these genes we calculated a literature neighbor score as well, by also including the relations of dexamethasone and inflammation with genes to which the gene is connected in the network. In Figure 3 it is shown that many genes which are not directly connected to inflammation (grey dots) are definitely influenced by inflammation via their connecting genes (black dots). 
The majority of the genes in Figure 3 are directly involved in important metabolic processes such as gluconeogenesis (PCK2, G6PC, PC and GCG), glycolysis (GCK, $G C G)$, glucose uptake, lipid metabolism (ACACA, CHPT1, GPD1) and carbohydrate metabolism (GPD1). Other ones are directly involved in insulin signaling (GIP, IGF2, IPF1, IAPP).

\section{Sex steroid physiology in relation to insulin resistance}

Interestingly in Figure 3 we also see three cytochrome P450s, i.e. CYP17A1, CYP19A1 and CYP21A2, which are key regulator enzymes in the steroid synthesis (Figure 4). The sub-network in Figure $2 \mathrm{C}$ shows the three cytocromes P450s and their direct gene neighbors. Analysis of this sub-network showed that many of the genes in the network are mentioned in studies from women suffering of the Polycystic ovary syndrome (PCOS), in which there is an imbalance of a woman's female sex hormones. PCOS is characterized by insulin resistance, possibly because of hyperandrogenism and low levels of SHBG. The latter effect has also been observed in men suffering from the metabolic syndrome [41]. Also a study by Macut et al. suggested that alterations of a cross-talk between glucocorticoid signaling and metabolic parameters, is related to PCOS pathophysiology [42].

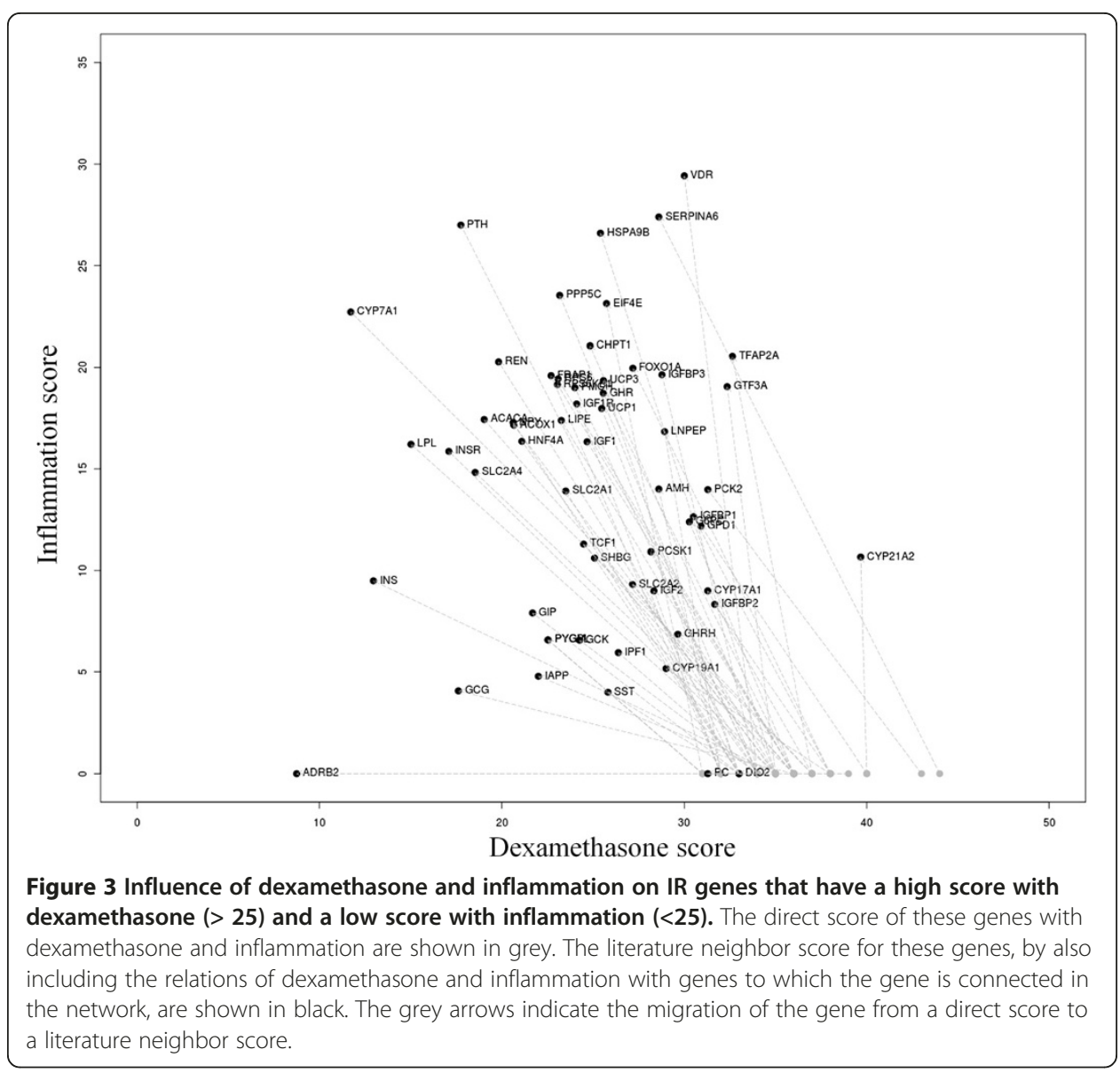




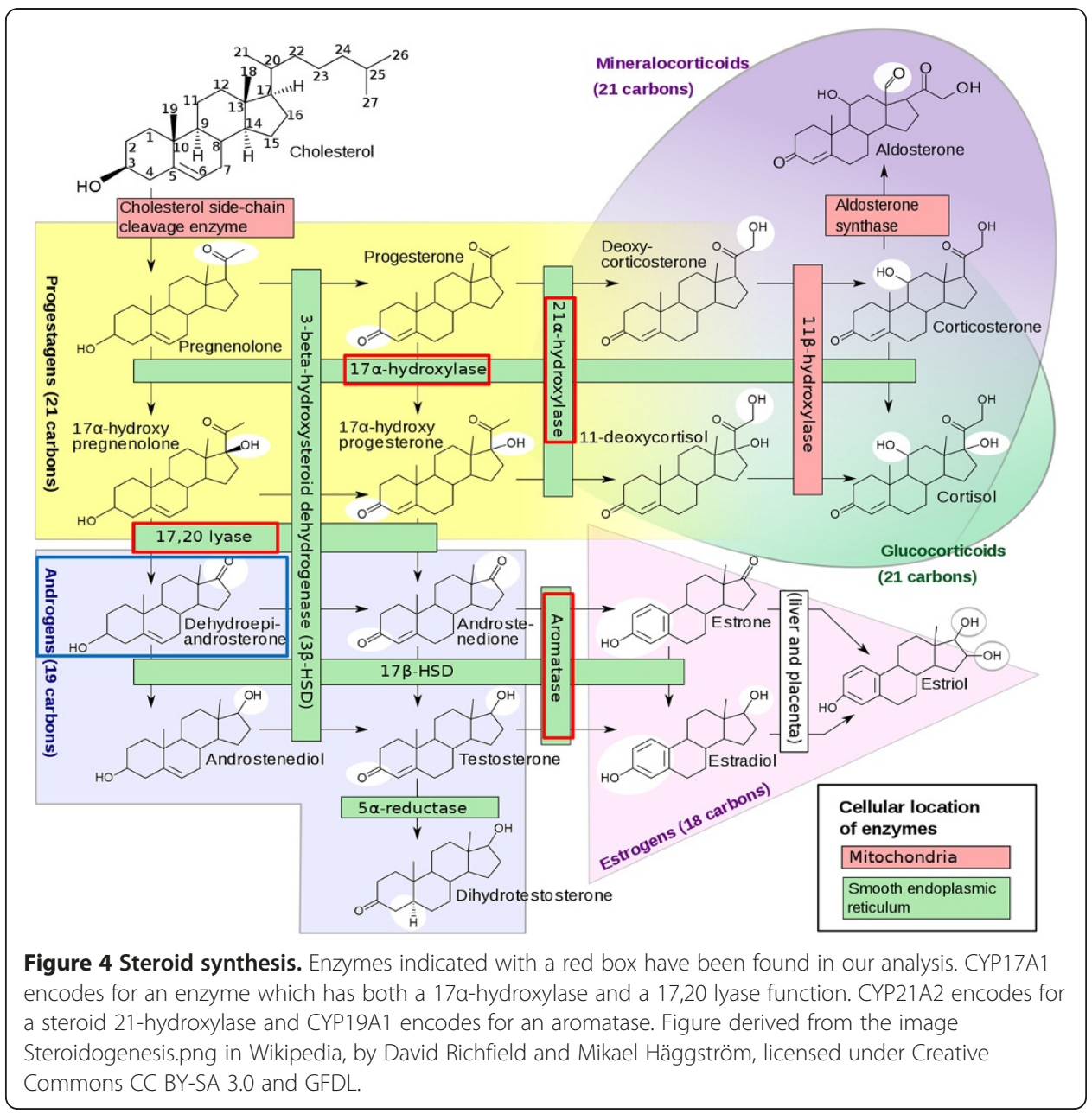

Additional topological analysis of the sub-network using cytohubba [43] revealed that IGF1R, HSD11B2, IGF2 and SHBG have a high betweenness centrality, i.e. they have many shortest paths going through them, analogous to major bridges and tunnels on a high map. Studies show that such a bottle necks play important roles in biological networks $[44,45]$.

CYP19A1 encodes for an aromatase which is responsible for the aromatization of androgens into estrogens, thus influencing the androgen to estrogen balance. Several studies showed that an imbalance between androgen and estrogen balance because of aromatase deficiency resulted in the development of symptoms related to the metabolic syndrome [46-49]. The fact that dexamethasone can regulate aromatase activity [50-52], suggests a role of aromatase in GC induced IR.

CYP17A1 is a key regulator of androgen synthesis and catalyzes the reactions in which pregnenolone and progesterone are converted into their 17-alpha-hydroxylated products and subsequently into Dehydroepiandrosterone (DHEA). A decline in DHEA and also its sulfated ester (DHEA-S) has been suggested to be causally linked to insulin resistance and obesity [53-56]. The possible inhibitory effects of dexamethasone on Cyp17a1 [57,58] suggests a role in GC induced IR by this gene.

CYP21A2 is a cytochrome P450 enzyme coding for the 21-hydroxylase that is involved in the biosynthesis of the steroid hormones aldosterone and cortisol. A defect 
in this gene leads to Congenital adrenal hyperplasia $(\mathrm{CAH})$ in which there is a disbalance in cortisol and aldosterone secretion. $\mathrm{CAH}$ patients are characterized by insulin resistance, lower insulin sensitivity and hyperinsulinemia [43,59-61]. Some studies indicate that the development of IR is because of GC treatment in this patient group [62-64]. Whether these patients develop IR because of CAH and deficiency of 21-hydroxylase, or because of the fact that they are often treated with synthetic GCs need to be elucidated.

\section{Genes involved in osteoporosis}

Another side effect of GC treatment is the development of glucocorticoid induced osteoporosis (GIOP) [65]. GIOP is characterized by reduced bone mineral density (BMD), decreased bone mass and disturbance of the bone matrix, leading to increased susceptibility to fractures. We applied CoPubGene to deduce important genes involved in GIOP by analyzing top scoring genes with OP (in total 131 genes associated with OP were found, see Additional file 2: Table S5; the network of these top scoring genes with relations to dexamethasone and inflammation is shown in Additional file 6: Figure S3. The majority of the genes are involved in bone remodeling and resorption (TNFRSF11A, TNFRSF11B, TNFSF11, SP7, CTSK), in bone mineralization (PTH, Klotho, VDR, Calca, BGLAP) or are part of the wnt signaling pathway that is involved in the regulation of bone formation (SOST, DKK1, LRP5, LRP6) [66]. Among these genes are known biomarkers of GIOP such as osteoprotegerin (encoded by TNFRSF11B) and the ligand RANK-L (encoded by TNFS11) [67]. Here we also searched for genes with a low score with inflammation. Several of these genes in the set, such as BGLAP, COL1A1 and SP7 are affected by GCs [68-72], have low associations with inflammation and therefore are interesting biomarker candidates for GIOP.

\section{Discussion}

In the work presented here we used Medline abstracts to study mechanisms and genes involved in glucocorticoid induced insulin resistance. We created CoPubGene, a number of web service operations that can be used to retrieve relevant gene-disease, genedrug and gene-gene associations out of Medline abstracts, using the CoPub technology.

The clustering of disease terms based on their associations with genes in Medline abstracts showed that CoPubGene is able to generate a list of specific IR genes that can be used for further analysis, and that this method also can be used to generate a variety of other gene disease associations. We used this clustering to evaluate the quality of disease related gene lists, generated using a text mining approach, because to our knowledge there is no real gold standard data set that covers a sufficient range of genedisease associations that can be used. Databases such as OMIM and the KEGG disease database [73] only cover a sub range of diseases which makes these datasets difficult to use in this type of evaluation.

Next, we studied the IR genes in their functional context, by including genes with which they co-occur in Medline abstracts. In this gene network we focused on genes that are strongly linked to dexamethasone and less strongly to inflammation. These genes are thought to be more exclusively related to GC induced IR and therefore might be interesting markers for this effect. 
However, all of them are to a certain extent related to inflammation, either directly or indirectly by their neighbors, which suggests that these genes cannot be used as an exclusive marker for GC induced IR. This might have consequences for the search of dissociating compounds, i.e. compounds which only have the immune suppressive properties and not the unwanted side effects. Instead the search should focus on compounds that show a reduced effect on the expression of these IR genes.

The majority of the IR genes that have a low literature neighbor score for inflammation $(<25)$ and a high score for dexamethasone (literature neighbor score $>25$ ) code for enzymes and hormones directly involved in important metabolic processes, such as glycolysis, gluconeogenesis, glucose uptake and lipid metabolism. All these processes are tightly regulated by insulin. This suggests that at a first instance, the search for mechanisms of GC induced IR should be focused on these processes.

Additionally, we also identified a sub network of genes involved in sex steroid synthesis that to our knowledge, not have been recognized yet as mediators of GC induced side effects. Key enzymes involved in steroid synthesis, i.e. CYP17A1, CYP21A2 and CYP19A1 keep the balance between several steroids, and an impairment of this balance could possibly result in metabolic disturbances such as IR. Additional topological analyses could further prioritize this sub-network for follow-up studies to determine the influence of GCs on sex steroid synthesis and the relation to IR. In such a study one could look at the influence of GCs on the balance between the steroids in combination with their influence on insulin stimulated glucose uptake in glucose sensitive tissues such as adipose and muscle tissue.

\section{Conclusions}

Using CoPubGene we are able to construct an informative literature network of IR related genes by only using information from Medline abstracts. Our approach revealed genes, that on a first glance were not considered to be involved in GC induced IR and thus gave new insights that might lead to a better understanding of the mechanisms behind GC induced IR.

\section{Additional files}

Additional file 1: Table S2. Part of the disease matrix, which has been used for the clustering.

Additional file 2: Table S2. 384 genes that are linked with insulin resistance in Medline abstracts. Table S3. Gene disease profiles. Table S4A. Enriched drug terms. Table S4B. Enriched disease terms. Table S4A.2. Genes linked with enriched drug terms. Table S4B.2. Genes linked with enriched disease terms. Table S5. 131 genes that linked with osteoporosis.

Additional file 3: Figure S2. Hierarchical cluster of disease terms from the CoPub database with bootstrapping values. Red numbers at the nodes represent Approximately Unbiased (AU) bootstrap values (\%). Green numbers at the nodes represent Bootstrap Probability (BP) value (\%).

Additional file 4: Enriched disease terms found per sub-cluster when searching with the DAVID annotation server.

Additional file 5: Figure S1. Distribution of connectivity of IR related gene network. The node connectivity follows a significant power law distribution ( $p$-value <0.001)

Additional file 6: Figure S3. Network of top scoring genes with osteoporosis. Genes in blue have a cooccurrence with dexamethasone in Medline abstracts (R-scaled score). The strength of the link with dexamethasone is given by the color shading, ranging from no link (white) to a strong link (dark blue). The strength of the link with inflammation (R-scaled score) is given by the size of the node of the gene, ranging from no link (normal size of the node) to a strong link with inflammation (large size of the node). 


\section{Authors' contributions}

WF performed all data analysis, research design and wrote the paper. ET supervised with biological interpretation and helped with writing the paper. SV, TH developed the web service operations. TR, RVS, RF and JDV helped with research design. WA supervised the work and helped with research design, analyzing the data and writing the manuscript. All authors read and approved the final manuscript.

\section{Financial contribution}

Author WF was supported by the Biorange project (BR4.2) "A Systems Bioinformatics Approach For Evaluating And Translating Drug-Target Effects In Disease Related Pathways" of NBIC.

\section{Author details}

${ }^{1}$ Computational Drug Discovery (CDD), CMBI, NCMLS, Radboud University Nijmegen Medical Centre, P.O. Box 91016500 HB, Nijmegen, The Netherlands. ${ }^{2}$ Netherlands Bioinformatics Centre (NBIC), P.O. Box 91016500 HB, Nijmegen, The Netherlands. ${ }^{3}$ Department of Medicine, Radboud University Nijmegen Medical Centre, Nijmegen, The Netherlands. ${ }^{4}$ Netherlands eScience Center, Amsterdam, The Netherlands. ${ }^{5}$ TNO, Zeist, The Netherlands. ${ }^{6}$ Present address: Rijk Zwaan Nederland BV, Fijnaart, The Netherlands. ${ }^{7}$ Present address: Philips Research Europe, Eindhoven, The Netherlands.

${ }^{8}$ Present address: NIZO Food Research BV, Ede, The Netherlands.

Received: 8 June 2012 Accepted: 2 January 2013

Published: 4 February 2013

\section{References}

1. Del Rosso Do JQ: Combination topical therapy for the treatment of psoriasis. J Drugs Dermato/ 2006, 5(3):232-234

2. Schwartz M, Cohen R: Optimizing conventional therapy for inflammatory bowel disease. Curr Gastroenterol Rep 2008, 10(6):585-590.

3. Hillier SG: Diamonds are forever: the cortisone legacy. J Endocrinol 2007, 195(1):1-6.

4. De Bosscher K, Haegeman G: Minireview: latest perspectives on antiinflammatory actions of glucocorticoids. Mol Endocrinol 2009, 23(3):281-291.

5. Rhen T, Cidlowski JA: Antiinflammatory action of glucocorticoids-new mechanisms for old drugs. N EnglJ Med 2005, 353(16):1711-1723

6. Rockall $A G$, et al: Computed tomography assessment of fat distribution in male and female patients with Cushing's syndrome. Eur J Endocrinol 2003, 149(6):561-567.

7. Schacke H, Docke WD, Asadullah K: Mechanisms involved in the side effects of glucocorticoids. Pharmacol The 2002, 96(1):23-43.

8. Schacke $\mathrm{H}$, et al: Insight into the molecular mechanisms of glucocorticoid receptor action promotes identification of novel ligands with an improved therapeutic index. Exp Dermatol 2006, 15(8):565-573.

9. Diamond $\mathrm{Ml}$, et al: Transcription factor interactions: selectors of positive or negative regulation from a single DNA element. Science 1990, 249(4974):1266-1272.

10. Schenk S, Saberi M, Olefsky JM: Insulin sensitivity: modulation by nutrients and inflammation. J Clin Invest 2008, 118(9):2992-3002.

11. Kalupahana NS, Moustaid-Moussa N, Claycombe KJ: Immunity as a link between obesity and insulin resistance. Mol Aspects Med 2012, 33(1):26-34.

12. Weinstein SP, et al: Dexamethasone inhibits insulin-stimulated recruitment of GLUT4 to the cell surface in rat skeletal muscle. Metabolism 1998, 47(1):3-6.

13. Ideker T, Lauffenburger D: Building with a scaffold: emerging strategies for high- to low-level cellular modeling. Trends Biotechnol 2003, 21(6):255-262.

14. Alkema W, Rullmann T, van Elsas A: Target validation in silico: does the virtual patient cure the pharma pipeline? Expert Opin Ther Targets 2006, 10(5):635-638.

15. Sharan R, Ideker T: Modeling cellular machinery through biological network comparison. Nat Biotechnol 2006, 24(4):427-433.

16. Goh Kl, et al: The human disease network. Proc Natl Acad Sci U S A 2007, 104(21):8685-8690.

17. Plake C, Schroeder M: Computational polypharmacology with text mining and ontologies. Curr Pharm Biotechnol 2011, 12(3):449-457.

18. Alako BT, et al: CoPub Mapper: mining MEDLINE based on search term co-publication. BMC Bioinformatics 2005, 6:51.

19. Frijters R, et al: CoPub: a literature-based keyword enrichment tool for microarray data analysis. Nucleic Acids Res 2008, 36(Web Server issue):W406-410.

20. Fleuren WW, et al: CoPub update: CoPub 5.0 a text mining system to answer biological questions. Nucleic Acids Res 2011, 39(Web Server issue):W450-454

21. Friberg PA, Larsson DG, Billig H: Transcriptional effects of progesterone receptor antagonist in rat granulosa cells. Mol Cell Endocrinol 2010, 315(1-2):121-130

22. Frijters $\mathrm{R}$, et al: Prednisolone-induced differential gene expression in mouse liver carrying wild type or a dimerization-defective glucocorticoid receptor. BMC Genomics 2010, 11:359.

23. Frijters $\mathrm{R}$, et al: Literature-based compound profiling: application to toxicogenomics. Pharmacogenomics 2007 8(11):1521-1534

24. Merkl M, et al: Microarray analysis of equine endometrium at days 8 and 12 of pregnancy. Biol Reprod 2010, 83(5):874-886

25. Mitterhuemer $S$, et al: Escherichia coli infection induces distinct local and systemic transcriptome responses in the mammary gland. BMC Genomics 2010, 11:138 
26. Shimizu T, et al: Actions and interactions of progesterone and estrogen on transcriptome profiles of the bovine endometrium. Physiol Genomics 2010, 42A(4):290-300.

27. Voice MW, Webster AP, Burchell A: The in vivo regulation of liver and kidney glucose-6-phosphatase by dexamethasone. Horm Metab Res 1997, 29(3):97-100

28. Franckhauser $S$, et al: Expression of the phosphoenolpyruvate carboxykinase gene in 3T3-F442A adipose cells: opposite effects of dexamethasone and isoprenaline on transcription. Biochem J 1995, 305(Pt 1):65-71.

29. Huson DH, et al: Dendroscope: An interactive viewer for large phylogenetic trees. BMC Bioinformatics 2007, 8:460.

30. Huang da W, Sherman BT, Lempicki RA: Systematic and integrative analysis of large gene lists using DAVID bioinformatics resources. Nat Protoc 2009, 4(1):44-57.

31. Huang da W, Sherman BT, Lempicki RA: Bioinformatics enrichment tools: paths toward the comprehensive functional analysis of large gene lists. Nucleic Acids Res 2009, 37(1):1-13.

32. Barabasi AL: Scale-free networks: a decade and beyond. Science 2009, 325(5939):412-413.

33. Xu H, et al: Dual specificity MAPK phosphatase 3 activates PEPCK gene transcription and increases gluconeogenesis in rat hepatoma cells. J Biol Chem 2005, 280(43):36013-36018.

34. Park JJ, et al: GRB14, GPD1, and GDF8 as potential network collaborators in weight loss-induced improvements in insulin action in human skeletal muscle. Physiol Genomics 2006, 27(2):114-121.

35. Krempler F, et al: Leptin, peroxisome proliferator-activated receptor-gamma, and CCAAT/enhancer binding protein-alpha mRNA expression in adipose tissue of humans and their relation to cardiovascular risk factors. Arterioscler Thromb Vasc Biol 2000, 20(2):443-449.

36. Chia YY, et al: Amelioration of glucose homeostasis by glycyrrhizic acid through gluconeogenesis rate-limiting enzymes. Eur J Pharmacol 2012, 677(1-3):197-202.

37. Fernandez-Real $\mathrm{JM}$, et al: Serum corticosteroid-binding globulin concentration and insulin resistance syndrome: a population study. J Clin Endocrinol Metab 2002, 87(10):4686-4690.

38. Kern PA, et al: Adipose tissue tumor necrosis factor and interleukin-6 expression in human obesity and insulin resistance. Am J Physiol Endocrinol Metab 2001, 280(5):E745-751.

39. Weisberg SP, et al: CCR2 modulates inflammatory and metabolic effects of high-fat feeding. J Clin Invest 2006, 116(1):115-124.

40. Tamura $Y$, et al: C-C chemokine receptor 2 inhibitor improves diet-induced development of insulin resistance and hepatic steatosis in mice. J Atheroscler Thromb 2010, 17(3):219-228.

41. Bhasin $\mathrm{S}$, et al: Sex hormone-binding globulin, but not testosterone, is associated prospectively and independently with incident metabolic syndrome in men: the framingham heart study. Diabetes Care 2011, 34(11):2464-2470.

42. Macut D, et al: Age, body mass index, and serum level of DHEA-S can predict glucocorticoid receptor function in women with polycystic ovary syndrome. Endocrine 2010, 37(1):129-134.

43. Mooij CF, et al: Unfavourable trends in cardiovascular and metabolic risk in paediatric and adult patients with congenital adrenal hyperplasia? Clin Endocrinol (Oxf) 2010, 73(2):137-146.

44. Yu H, et al: The importance of bottlenecks in protein networks: correlation with gene essentiality and expression dynamics. PLoS Comput Biol 2007, 3(4):e59.

45. McDermott JE, et al: Bottlenecks and hubs in inferred networks are important for virulence in Salmonella typhimurium. J Comput Biol 2009, 16(2):169-180.

46. Bader $\mathrm{Ml}$, et al: Comparative assessment of estrogenic responses with relevance to the metabolic syndrome and to menopausal symptoms in wild-type and aromatase-knockout mice. J Steroid Biochem Mol Biol 2011 $127((3-5)): 428-34$.

47. Jones ME, et al: Of mice and men: the evolving phenotype of aromatase deficiency. Trends Endocrinol Metab 2006, 17(2):55-64.

48. Maffei $L$, et al: Dysmetabolic syndrome in a man with a novel mutation of the aromatase gene: effects of testosterone, alendronate, and estradiol treatment. J Clin Endocrinol Metab 2004, 89(1):61-70.

49. Takeda $\mathrm{K}$, et al: Progressive development of insulin resistance phenotype in male mice with complete aromatase (CYP19) deficiency. J Endocrinol 2003, 176(2):237-246.

50. Zhao H, et al: A novel promoter controls Cyp19a1 gene expression in mouse adipose tissue. Reprod Biol Endocrinol 2009, 7:37.

51. Simpson $E R$, et al: Estrogen formation in stromal cells of adipose tissue of women: induction by glucocorticosteroids. Proc Natl Acad Sci U S A 1981, 78(9):5690-5694.

52. Enjuanes $A$, et al: Regulation of CYP19 gene expression in primary human osteoblasts: effects of vitamin D and other treatments. Eur J Endocrinol 2003, 148(5):519-526.

53. Koga $\mathrm{M}$, et al: Serum dehydroepiandrosterone sulphate levels in patients with non-alcoholic fatty liver disease. Intern Med 2011, 50(16):1657-1661.

54. Kurzman ID, MacEwen EG, Haffa AL: Reduction in body weight and cholesterol in spontaneously obese dogs by dehydroepiandrosterone. Int J Obes 1990, 14(2):95-104.

55. Sanchez J, et al: Dehydroepiandrosterone prevents age-associated alterations, increasing insulin sensitivity. J Nutr Biochem 2008, 19(12):809-818.

56. Schriock ED, et al: Divergent correlations of circulating dehydroepiandrosterone sulfate and testosterone with insulin levels and insulin receptor binding. J Clin Endocrinol Metab 1988, 66(6):1329-1331.

57. Lee TC, Miller WL, Auchus RJ: Medroxyprogesterone acetate and dexamethasone are competitive inhibitors of different human steroidogenic enzymes. J Clin Endocrinol Metab 1999, 84(6):2104-2110.

58. Trzeciak WH, et al: Dexamethasone inhibits corticotropin-induced accumulation of CYP11A and CYP17 messenger RNAs in bovine adrenocortical cells. Mol Endocrinol 1993, 7(2):206-213.

59. Speiser PW, et al: Insulin insensitivity in adrenal hyperplasia due to nonclassical steroid 21-hydroxylase deficiency. J Clin Endocrinol Metab 1992, 75(6):1421-1424.

60. Paula FJ, et al: Androgen-related effects on peripheral glucose metabolism in women with congenital adrenal hyperplasia. Horm Metab Res 1994, 26(11):552-556. 
61. Saygili F, Oge A, Yilmaz C: Hyperinsulinemia and insulin insensitivity in women with nonclassical congenital adrenal hyperplasia due to 21-hydroxylase deficiency: the relationship between serum leptin levels and chronic hyperinsulinemia. Horm Res 2005, 63(6):270-274.

62. Kroese JM, et al: Pioglitazone improves insulin resistance and decreases blood pressure in adult patients with congenital adrenal hyperplasia. Eur J Endocrinol 2009, 161(6):887-894.

63. Charmandari E, et al: Children with classic congenital adrenal hyperplasia have elevated serum leptin concentrations and insulin resistance: potential clinical implications. J Clin Endocrinol Metab 2002, 87(5):2114-2120.

64. Bachelot A, et al: Long-term outcome of patients with congenital adrenal hyperplasia due to 21-hydroxylase deficiency. Horm Res 2007, 67(6):268-276.

65. den Uyl D, Bultink IE, Lems WF: Glucocorticoid-induced osteoporosis. Clin Exp Rheumatol 2011, 29(5 Suppl 68):S93-98.

66. Issack PS, Helfet DL, Lane JM: Role of Wnt signaling in bone remodeling and repair. HSS J 2008, 4(1):66-70.

67. Canalis E: Mechanisms of glucocorticoid-induced osteoporosis. Curr Opin Rheumatol 2003, 15(4):454-457.

68. Kauh $\mathrm{E}$, et al: Prednisone affects inflammation, glucose tolerance, and bone turnover within hours of treatment in healthy individuals. Eur J Endocrinol 2012, 166(3):459-467.

69. Eastell $R$, et al: Bone formation markers in patients with glucocorticoid-induced osteoporosis treated with teriparatide or alendronate. Bone 2010, 46(4):929-934.

70. Zheng HF, Spector TD, Richards JB: Insights into the genetics of osteoporosis from recent genome-wide association studies. Expert Rev Mol Med 2011, 13:e28.

71. Fu H, et al: Osteoblast differentiation in vitro and in vivo promoted by Osterix. J Biomed Mater Res A 2007, 83(3):770-778.

72. Advani $\mathrm{S}$, et al: Dexamethasone suppresses in vivo levels of bone collagen synthesis in neonatal mice. Bone 1997, 20(1):41-46.

73. Kanehisa M, et al: KEGG for linking genomes to life and the environment. Nucleic Acids Res 2008 , 36(Database issue):D480-484.

doi:10.1186/1756-0381-6-2

Cite this article as: Fleuren et al: Identification of new biomarker candidates for glucocorticoid induced insulin resistance using literature mining. BioData Mining 2013 6:2.

\section{Submit your next manuscript to BioMed Central and take full advantage of:}

- Convenient online submission

- Thorough peer review

- No space constraints or color figure charges

- Immediate publication on acceptance

- Inclusion in PubMed, CAS, Scopus and Google Scholar

- Research which is freely available for redistribution

Submit your manuscript at www.biomedcentral.com/submit 